\title{
Dinámica de claros artificiales en un bosque aprovechado en Misiones, noreste de Argentina
}

\author{
Nardia Maria Luján Bulfe ${ }^{1}$, Franklin Galvão², Afonso Figueiredo Filho², Patricio Mac Donagh ${ }^{3}$
}

\begin{abstract}
${ }^{1}$ Instituto Nacional de Tecnología Agropecuaria (INTA), Av. Libertador, 2472-3384, Montecarlo, Argentina, nbulfe@montecarlo.inta.gov.ar; ${ }^{2}$ Univeridade Federal do Paraná (UFPR), Departamento de Ciências Florestais, Av. Pref. Lothário Meissner, 900, Jardim Botânico, CEP 80210170, Curitiba, PR, Brasil, fgalvao@ufpr.br; afonso.figueiredo@pq.cnpq.br; ${ }^{3}$ Universidad Nacional de Misiones (UNaM), Bertoni, 124, CP 3380, Eldorado, Misiones Argentina Argentina, mdonagh@facfor.unam.edu.ar
\end{abstract}

\begin{abstract}
Resumen - Este trabajo evalúa el crecimiento diamétrico de especies arbóreas clasificadas en grupos ecológicos dentro de claros artificiales en un bosque estacional semideciduo aprovechado considerando dos sistemas de cosecha. El estudio fue desarrollado en la Reserva de Biosfera Yabotí, Misiones, Argentina. Se instalaron 15 parcelas permanentes, de cuatro hectáreas cada una, con un área efectiva de medición de una hectárea. Los tratamientos fueron tres: cosecha convencional, cosecha de impacto reducido y parcela testigo. Se registraron todos los ejemplares arbóreos mayores a $10 \mathrm{~cm}$ de diámetro a la altura del pecho (DAP) antes (1998) y después de la cosecha (2006). La riqueza florística alcanzó un número de 85 especies. Para el conjunto de especies el crecimiento diamétrico promedio fue de $0,41 \mathrm{~cm}_{\text {año }}{ }^{-1}$ en área de claro y de $0,31 \mathrm{~cm}$.año ${ }^{-1}$ en área testigo, siendo el promedio de crecimiento diamétrico de $0,36 \mathrm{~cm}$.año ${ }^{-1}$ para impacto reducido y $0,42 \mathrm{~cm}$ año $0^{-1}$ para cosecha convencional. En bosques con alta diversidad florística, se debería considerar a los grupos ecológicos como una herramienta base para el manejo y conservación de los mismos. El aprovechamiento de impacto reducido debería ser considerado como una alternativa menos agresiva para los bosques de la región de Misiones.
\end{abstract}

Términos para indexación: Grupos ecológicos, cosecha, bosque neotropical, crecimiento.

\section{Dynamics of treefall gaps in a harvested forest in Misiones, Northern Argentine}

\begin{abstract}
This work evaluates the diameter growth of trees species of artificial gaps per ecological groups in a harvested forest considering two systems of harvest. The study was carried out in the Reserva de Biósfera Yabotí, in Misiones, Argentina. Fifteen permanent plots were established, each one with 4 hectares with effective area of measurement of one hectare. The three treatments were: conventional logging, reduced impact logging and control plot. All trees larger than $10 \mathrm{~cm}$ of diameter at breast height (DBH) were measured, before (1998) and after the harvest (2006). Eighty-five species were registered. The average DBH diameter growth for all species was $0.41 \mathrm{~cm} \mathrm{yr}^{-1}$ in the gap area, and $0.31 \mathrm{~cm} \mathrm{yr}^{-1}$ for the control plot. The average diameter growth was $0.36 \mathrm{~cm} \mathrm{yr}^{-1}$ for the reduced impact logging and $0.42 \mathrm{~cm} \mathrm{yr}^{-1}$ for the conventional logging. In forests with high diversity, ecological groups are an important tool for the management and conservation. The reduced impact logging would be considered a less aggressive alternative for the forests in this region.
\end{abstract}

Index terms: ecological groups, logging, neotropical forest, growth.

\section{Introducción}

El bosque tiene un amplio espectro de microambientes en los cuales las especies reaccionan de modos diferentes, de esta manera nace el concepto de clasificación de especies en diferentes grupos ecológicos, siendo esta una de las principales bases ecológicas para el manejo de los bosques tropicales y subtropicales (Guzmán G., 1997).

Louman et al. (2001) mencionan que tradicionalmente se ha denominado como grupo ecológico al conjunto de especies que poseen características biológicas y ecológicas similares. Existen en la literatura muchas clasificaciones relativas a los grupos ecológicos (Budowski, 1965; Denslow, 1980; Whitmore, 1984; Swaine \& Whitmore, 1988). Según Finegan (1993), el propósito de clasificar a las especies en grupos más homogéneos, en cuanto a sus requerimientos ambientales, es producir una simplificación de la información, revelando padrones generales y facilitando las predicciones del proceso del bosque. 
Según Scatena (2002), la vegetación que se desarrolla en un determinado paisaje está influenciada por tres factores: la estructura geológica, los gradientes ambientales y los regímenes de perturbaciones naturales. Connell (1978) propone la hipótesis de las perturbaciones intermediarias donde, en composición de especies, si una comunidad no alcanza el equilibrio y la alta diversidad eso se debe a un constante cambio en las condiciones del ecosistema.

Las aberturas provocadas en el dosel crean un ambiente diferente de su entorno, estando estrechamente relacionado al tamaño del claro (Hogan \& Machado, 2002; Ferreira Lima, 2005). De esta manera los claros adquieren importancia cuando se estudia la dinámica del bosque. Así, la conjunción entre grupos ecológicos y estructura de claros pasa a ser una herramienta interesante para generar información en relación la la dinámica del bosque.

En la dinámica arbórea de un bosque deben ser considerados tres componentes: incremento diamétrico, mortalidad y reclutamiento (Finegan, 1993). Uhl et al. (1988) determinaron que el crecimiento de los individuos arbóreos ( $\geq 10 \mathrm{~cm}$ DAP) es influenciado por: tipo de micro-hábitat, grupo ecológico, tamaño del individuo, tamaño y edad del claro. Los mismos autores determinaron que la probabilidad de muerte de un individuo disminuye según su diámetro se incrementa.

La escala regional, un factor de suma importancia en la dinámica de los bosques en áreas disturbadas, es la colonización de manera agresiva por bambúseas (Merostachys y Chusquea). Campanello (2004) encontró que la radiación fotosintéticamente activa incidente a 0,7 metros sobre el suelo fue menor que a 2 metros en un área con presencia de bambúseas.

Rivero et al. (2008) determinaron que el crecimiento diamétrico entre tratamientos no presentó diferencias estadísticas significativas cuatro años después del aprovechamiento. Desde el punto de vista de los daños ocasionados a los remanentes, se destacó una reducción de los mismos mediante la aplicación de la cosecha de impacto reducido (Mac Donagh et al., 2003). Estos autores encontraron una relación positiva entre la intensidad de cosecha y el área basal dañada, y destacan al daño a la copa como el de mayor importancia, lo cual afecta el crecimiento diamétrico de los individuos posteriormente a la cosecha.

El objetivo de este trabajo fue evaluar la dinámica de especies arbóreas por grupos ecológicos en claros artificiales originados a partir de dos técnicas de cosecha distintas, convencional y de impacto reducido siete años después de la intervención.

\section{Material y métodos}

\section{Área de estudio}

El presente estudio fue desarrollado en la Reserva Forestal de Uso Múltiple Guaraní (5.343 hectáreas), localizada dentro de la Reserva de Biosfera Yabotí 26 $6^{\circ} 57^{\text {' }}$ Sur y $54^{\circ} 15^{\prime}$ Oeste, en Misiones, Argentina. La altimetría presenta las cotas extremas de $267 \mathrm{~m}$ y $574 \mathrm{~m}$, donde un $50 \%$ de la superficie está comprendida entre 400 y 500 m (Palavecino \& Maiocco, 1995). El clima de la región corresponde, según Köppen, al tipo Cfa, siendo subtropical mesotérmico sin estación seca. La precipitación media anual es de $1.800 \mathrm{~mm}$. La temperatura media anual del aire es de $21^{\circ} \mathrm{C}$ (Eibl et al., 1995). Los suelos corresponden, según taxonomía americana, a los órdenes: Inceptisoles (31\%), Entisoles (20\%), Molisoles (18\%), Alfisoles (13,4\%), Ultisoles (8\%) y Oxisoles $(6,6 \%)$; y el $3 \%$ pertenece a áreas con presencia de afloramientos de roca (Pahr et al., 1997). Por el sistema brasileño de clasificación de suelos serían respectivamente: Cambissolos, Neossolos, Chernossolos, Luvissolos, Nitossolos e Latossolos.

El área de estudio está cubierta por bosque estacional semideciduo, conformado por diferentes estratos. Tressens \& Revilla (1997) encontraron, en un área próxima, 114 especies arbóreas, distribuidas en 92 géneros correspondientes a 42 familias.

\section{Descripción del ensayo}

Fueron instaladas 15 parcelas permanentes de 4 hectáreas cada una (200 m x $200 \mathrm{~m})$. Cada parcela cuenta con un área central de medición de una hectárea, con una zona de amortiguación de $50 \mathrm{~m}$ a cada lado (Fig. 1). Las parcelas fueron sometidas a diferentes metodologías de cosecha, lo cual resultó en los tres tratamientos: cosecha de impacto reducido (CIR): basado en la planificación y control de la implementación de las operaciones de cosecha, minimizando el impacto sobre el remanente. cosecha convencional (CC): todas las operaciones de la cosecha fueron determinadas por el operario de la maquinaria al momento del aprovechamiento. Parcelas testigo (PT): área no aprovechada, sin tránsito de maquinarias. 
La cosecha se realizó durante los meses de junio y julio del año 1999, donde fueron talados dentro de la hectárea de medición, en los diferentes tratamientos, 45 individuos en las 12 parcelas con intervención convencional y de impacto reducido, estos individuos estuvieron distribuidos entre 11 especies diferentes.

La intensidad promedio de cosecha para el ensayo en general fue de 3,75 ind ha-1 $(\mathrm{SD}=2,10 ; \mathrm{N}=45)$, siendo de 5,2 ind $\mathrm{ha}^{-1}(\mathrm{SD}=2,20 ; \mathrm{N}=26)$ para cosecha convencional y 2,70 ind ha $^{-1}(\mathrm{SD}=1,30 ; \mathrm{N}=19)$ para cosecha de impacto reducido. Los tratamientos crearon una superficie total de claros de $8.018,7 \mathrm{~m}^{2}$, representando $6,7 \%$ de un área de muestreo de $120.000 \mathrm{~m}^{2}$, donde $65 \%$ del área de claros fue creada por CC (Bulfe et al., 2009).

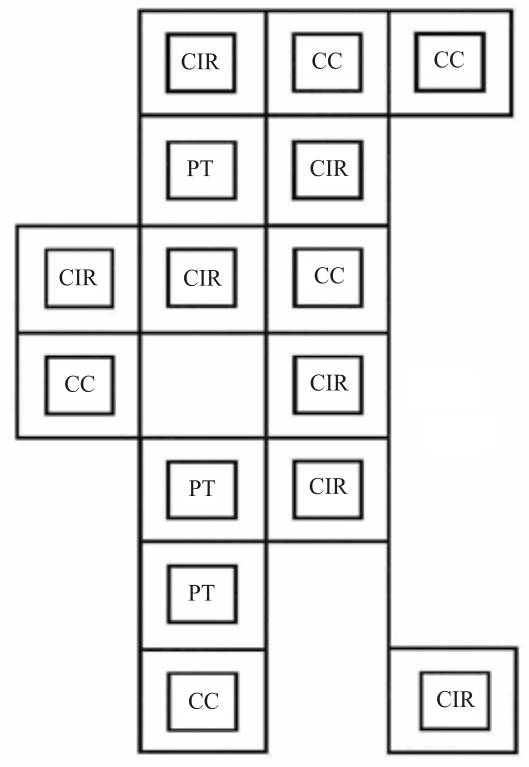

Figura 1. Diseño experimental del área de estudio, Reserva de Uso Múltiple Guaraní, Misiones, Argentina. CC: Cosecha convencional, CIR: Cosecha de impacto reducido, PT: Parcela testigo.

Los individuos fueron cosechados llevando en consideración el diámetro mínimo de corte (DMC) determinado por el Ministerio de Ecología de la provincia de Misiones, según el cual cada individuo perteneciente a una especie establecida en la lista de las comerciales debe presentar un diámetro mínimo comprendido entre $35 \mathrm{~cm}$ y $55 \mathrm{~cm}$, a partir del cual las diferentes especies pueden ser taladas.

Para evaluar la dinámica de las especies establecidas en el área de claro, se procedió a la localización de la cepa del árbol extraído, donde cada individuo apeado conformó un claro artificial, tomando a la altura total del árbol apeado (datos registrados antes del aprovechamiento, en el año 1998) como el radio del claro, siendo ésta denominada como "área de claro". Todos los individuos dentro de esta área de claro fueron considerados en la evaluación de la dinámica. De esa forma, se delimitaron 45 claros artificiales en ambos tratamientos, cosecha convencional y de impacto reducido. Como referencia para la comparación fueron utilizados todos los individuos arbóreos relevados en las tres parcelas de una hectárea de cada una de las parcelas testigo.

A los fines de cumplir con el objetivo planteado en el presente trabajo, se tomarán en cuenta las siguientes variables: incremento diamétrico, ingreso y mortalidad, riqueza y diversidad florística.

\section{Obtención de datos y establecimiento de criterios}

Para la determinación del incremento diamétrico se consideraron todos los ejemplares arbóreos mayores o iguales a $10 \mathrm{~cm}$ de DAP dentro del área de claro antes (1998) y siendo remedidos siete años después de la cosecha (2006). Todos los árboles se identificaron a nivel de especie.

Para la determinación de los ingresos arbóreos en la dinámica del bosque fueron considerados los individuos que alcanzaron o superaron los 10 de DAP dentro del área de claro durante el período de medición.

Debido a que en la zona se carece de información con respecto a las características y comportamiento para la mayor parte de las especies nativas, para la clasificación de las mismas en grupos ecológicos se debió realizar una clasificación tomando como base información disponible en bibliografías como libros de Carvalho (2003-2008) y de fichas técnicas publicadas en diferentes revistas Yvyraretá, las cuales pertenecen a estudios realizados sobre características morfológicas y ecológicas de las principales especies de Misiones y del sur de Brasil. Así, que se optó por utilizar una clasificación basada en Swaine \& Whitmore (1988), junto con la experiencia de los autores, donde se consideraron los siguientes criterios: velocidad de crecimiento, color y densidad de la madera, tamaño y cantidad de semillas, características de las hojas, etapa de la sucesión en la que predomina y posición sociológica en el dosel. Los grupos ecológicos fueron cinco y la nomenclatura utilizada para ello fue la siguiente (Tabla 1): 
Tabla 1. Grupos ecológicos de las especies evaluadas.

\begin{tabular}{|c|c|c|c|c|c|}
\hline \multirow{2}{*}{ Nombre científico } & \multicolumn{5}{|c|}{ Grupos ecológicos } \\
\hline & $\mathbf{P}$ & P/CEL & CEL/P & CEL & CS \\
\hline Ateleia glazioveana Baill. & 1 & & & & \\
\hline Bastardiopsis densiflora Hassler & 1 & & & & \\
\hline Inga marginata Willd. & 1 & & & & \\
\hline Styrax leprosum Hook. \& Arn. & 1 & & & & \\
\hline Dendropanax cuneatus Decne. \& Planch. & & 1 & & & \\
\hline Didymopanax morototoni Decne. \& Planch. & & 1 & & & \\
\hline Chorisia speciosa A. St. -Hill. & & 1 & & & \\
\hline Cordia trichotoma (Vell.) Steud. & & 1 & & & \\
\hline Dalbergia variabilis Vogel & & 1 & & & \\
\hline Fagara hiemalis Engl. & & 1 & & & \\
\hline Lonchocarpus leucanthus Burkart & & 1 & & & \\
\hline Lonchocarpus muehlbergianus Hassler & & 1 & & & \\
\hline Luehea divaricata Mart. & & 1 & & & \\
\hline Machaerium brasiliense Vogel & & 1 & & & \\
\hline Matayba elaeagnoides Radlk. & & 1 & & & \\
\hline Ocotea puberula Nees & & 1 & & & \\
\hline Parapiptadenia rigida (Benth.) Brenan & & 1 & & & \\
\hline Patagonula americana $\mathrm{L}$. & & 1 & & & \\
\hline Sebastiania brasiliensis Spreng. & & 1 & & & \\
\hline Sebastiania commersoniana (Baill.) L.B.Sm. \& R.J.Downs & & 1 & & & \\
\hline Vitex megapotamica (Spreng.) Moldenke & & 1 & & & \\
\hline Balfourodendron riedelianum Engl. & & & 1 & & \\
\hline Campomanesia xanthocarpa O. Berg & & & 1 & & \\
\hline Cedrela fissilis Vell. & & & 1 & & \\
\hline Prunus subcoriacea Koehne & & & 1 & & \\
\hline Ruprechtia laxiflora Meisn. & & & 1 & & \\
\hline Apuleia leiocarpa J.F. Macbr. & & & & 1 & \\
\hline Aspidosperma australe Müll.Arg. & & & & 1 & \\
\hline Chrysophyllum gonocarpum Engl. & & & & 1 & \\
\hline Chrysophyllum marginatum Radlk. & & & & 1 & \\
\hline Diatenopteryx sorbifolia Radlk. & & & & 1 & \\
\hline Holocalyx glaziovii Taub. ex Glaz. & & & & 1 & \\
\hline Jacaratia spinosa A. D.C. & & & & 1 & \\
\hline Myrocarpus frondosus Allem. & & & & 1 & \\
\hline Nectandra megapotamica $\mathrm{Mez}$ & & & & 1 & \\
\hline Achatocarpus praecox Griseb. & & & & & 1 \\
\hline Allophylus edulis Radlk. ex Warm. & & & & & 1 \\
\hline Casearia decandra Jacq. & & & & & 1 \\
\hline Casearia sylvestris $\mathrm{Sw}$. & & & & & 1 \\
\hline Helietta apiculata Benth. & & & & & 1 \\
\hline Ocotea diospyrifolia (Meisn.) Mez & & & & & 1 \\
\hline Picrasma crenata Engl. & & & & & 1 \\
\hline Pilocarpus pennatifolius Lem. & & & & & 1 \\
\hline Sorocea bonplandii (Baill.) W.C.Burger, Lanj. \& Boer & & & & & 1 \\
\hline Strychnos brasiliensis Mart. & & & & & 1 \\
\hline Trichilia catigua A. Juss. & & & & & 1 \\
\hline Trichilia claussenii C. DC. & & & & & 1 \\
\hline
\end{tabular}

$\mathrm{P}=$ Pionera, $\mathrm{PCEL}=$ Pionera con tendencia a Climax Exigentes en Luz, CELP $=$ Climax Exigentes en Luz con tendencia a Pionera, $\mathrm{CEL}=$ Climax Exigentes en Luz, CS= Climax con Tolerancia a la Sombra. 
Pioneras (P) - especies que requieren de plena insolación durante toda su vida, de reproducción masiva y precoz, de crecimiento rápido y de vida relativamente corta;

Climax Exigentes en Luz (CEL) - especies que regeneran a la sombra (fase juvenil), pero que exigen altas intensidades de luz cuando alcanzan la madurez (fase adulta), posicionándose en el dosel del bosque, y son de vida relativamente larga;

Climax Tolerantes a la Sombra (CS) - especies que se regeneran a la sombra del dosel y son capaces de efectuar allí todo su desarrollo vegetativo y reproductivo;

Pioneras con tendencia a Climax Exigentes en Luz (PCEL): especies comunes a los dos grupos ecológicos, con características predominantes de Pioneiras;

Climax Exigentes en Luz con tendencia a Pioneras (CELP): especies comunes a los dos grupos ecológicos, con características predominantes de Climax Exigentes en Luz.

\section{Resultados y discusión}

\section{Composición florística}

Fueron identificadas un total de 85 especies distribuidas en 34 familias botánicas diferentes. En las parcelas testigo fueron identificadas 68 especies y en cada tratamiento: 73 especies en cosecha convencional (CC) y 65 en cosecha de impacto reducido (CIR). Del total de las especies determinadas en el ensayo, 58\% eran comunes a los tres tratamientos, siendo las principales, en cuanto a densidad Nectandra megapotamica (Spreng.) Mez, Lonchocarpus leucanthus (Burkart) N.F. Mattos, Balfourodendron riedelianum (Engl.) Engl. y Trichillia catigua A. Juss.

Los valores de diversidad de Shannon resultaron de 3,33 nats ind ${ }^{-1}$ en las parcelas testigo y 3,37 nats ind ${ }^{-1}$ para CC y 3,47 nats ind $^{-1}$ en CIR. El análisis de varianza no mostró diferencias estadísticamente significativas entre tratamientos para este parámetro (ANOVA, $p=0,780$ ). En este sentido el período de ocho años considerado (1998-2006, antes y después del aprovechamiento) tal vez haya sido escaso para detectar modificaciones en este parámetro.

La riqueza florística determinada en el área de estudio fue mayor a lo encontrado por Gauto (1997) en un área próxima a la estudiada en este trabajo. Este autor identificó un total de 72 especies arbóreas en 4 hectáreas sometidas a explotación forestal, siendo las especies con mayor número de individuos coincidentes con las mencionadas aquí, a excepción de T. catigua. La mayor riqueza podría estar relacionada al mayor tamaño de muestreo utilizado en el presente trabajo.

\section{Incremento diamétrico}

En las parcelas donde se realizó algún tratamiento de cosecha, el incremento diamétrico para el conjunto de especies establecidas dentro del área de claro fue de $0,42 \mathrm{~cm}^{2}$ año ${ }^{-1}(\mathrm{SD}=0,34 ; \mathrm{N}=47)$ mientras que en

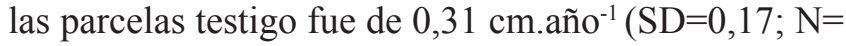
47). Rivero et al. (2008) encontraron igual tendencia en resultados de una comparación del incremento diamétrico de individuos creciendo en áreas con y sin aprovechamiento.

El 74\% de las especies establecidas en el área de claro presentaron incrementos diamétricos superiores cuando comparadas con las mismas especies en las parcelas testigo. Las más destacadas en los claros fueron: Jacaratia spinosa (Aubl.) A. DC., Bastardiopsis densiflora (Hook. \& Arn.) Hassl. y Luehea divaricata Mart., presentando éstas valores de incremento diamétrico superiores a $1 \mathrm{~cm}$ año ${ }^{-1}$. Estas especies, previo al análisis, fueron catalogadas en grupos ecológicos de rápido crecimiento en condiciones óptimas de luminosidad.

Las distribuciones de los incrementos diamétricos para áreas de claros y parcelas testigo fueron estadísticamente significativas (U Mann-Whitney: $Z=4,815 ; \mathrm{p}<0,05$ ). Los grupos ecológicos tuvieron incrementos diamétricos medios superiores en áreas de claros, a excepción del grupo ecológico CS, las cuales tuvieron un incremento diamétrico medio superior en parcelas testigo.

El incremento diamétrico medio de los individuos establecidos en áreas de claro bajo las diferentes modalidades de cosecha fueron de $0,36 \mathrm{~cm}$ año ${ }^{-1}$ para CIR ( $\mathrm{SD}=0,37 ; \mathrm{N}=47)$ y de $0,42 \mathrm{~cm}^{2}$ ño ${ }^{-1}$ para $\mathrm{CC}$ $(\mathrm{SD}=0,32 ; \mathrm{N}=47)$. Las distribuciones de los incrementos diamétricos de los individuos bajo las diferentes modalidades de cosecha fueron estadísticamente significativas ( $U$ Mann-Whitney: $Z=3,063 ; p<0,05$ ), coincidiendo con lo determinado por Rivero et al. (2008) cuando comparan los incrementos diamétricos de las especies creciendo en áreas donde fueron aplicados diferentes modalidades de aprovechamiento (convencional e impacto reducido).

En un área cercana al área de estudio de este trabajo, Gauto (1997) determinó un incremento promedio, para el conjunto de especies, de $0,58 \mathrm{~cm}_{\text {año }}{ }^{-1}$ en una parcela permanente de cuatro hectáreas sometida a cosecha 
selectiva con una intensidad promedio de cosecha de 17,5 ind ha ${ }^{-1}$, lo cual estaría explicando el mayor crecimiento. Sin embargo, Sist \& Ferreira (2007), con una intensidad promedio de cosecha de seis ind $\mathrm{ha}^{-1}$, determinaron un crecimiento diamétrico promedio de $0,4 \mathrm{~cm}$ año ${ }^{-1}$ para un bosque en el Amazona sometido también a cosecha selectiva.

Las áreas de claros creadas bajo el tratamiento de CIR presentaron una mayor proporción de número de individuos de especies enmarcadas en los grupos ecológicos CS y PCEL, mientras que en claros del tratamiento $\mathrm{CC}$ hubo una mayor proporción de individuos de los grupos ecológicos CEL y PCEL. En el ensayo fue observado por Bulfe et al. (2009) una cobertura de dosel mayor en las parcelas testigo cuando comparadas con las parcelas donde se aplicó algún tratamiento de cosecha. De esta forma, en función de la modalidad de cosecha se generan variados microambientes debido a diferentes intensidades lumínicas, favoreciendo así a las especies de grupos ecológicos.

Si bien no existe información disponible sobre la dinámica de grupos ecológicos para el tipo de bosque en la región de estudio, con base en el conocimiento de las características ecológicas de las especies estudiadas, la dinámica de los grupos ecológicos presentaron un comportamiento lógico y esperado (Tabla 2), principalmente para las del grupo ecológico CS que demostraron ser mas eficientes en ambientes de menor luminosidad como lo son las áreas de las parcelas testigo y en claros ocasionados por CIR, pudiendo estar relacionado a los valores de cobertura del dosel.

Tabla 2. Media del incremento diamétrico $(\mathrm{cm}$ año-1) por grupos ecológicos, para el período 1998-2006, en áreas de claro y parcelas testigo.

\begin{tabular}{lcc}
\hline \multirow{2}{*}{$\begin{array}{l}\text { Grupos } \\
\text { ecológicos }\end{array}$} & Claros & \multicolumn{1}{c}{ Parcelas testigo } \\
\cline { 2 - 3 } P & \multicolumn{2}{c}{$\mathbf{( c m ~}$ año $\left.^{-1}\right)$} \\
PCEL & 0,72 & 0,46 \\
CELP & 0,33 & 0,25 \\
CEL & 0,50 & 0,40 \\
CS & 0,35 & 0,26 \\
\hline
\end{tabular}

Al estudiar el crecimiento por grupos ecológicos se pudo observar una homogenización de los datos, notando una tendencia marcada entre los tres grupos conformados por especies con características más definidas como $\mathrm{P}$, CEL y CS, mientras que los otros grupos (PCEL y CELP) aportarían la mayor variabilidad en los datos cuando se pretende estudiarlas en la composición florística toda. De esta manera, y coincidiendo con otros autores como Finegan (1993), Guzmán G. (1997) y Martins-Maciel et al. (2002), el uso de los grupos ecológicos debería ser considerado una herramienta para el manejo de los bosques con alta riqueza específica.

Varios factores intervienen en la variabilidad del crecimiento, donde los principales resultan ser la disponibilidad de luz, competencia, variabilidad genética (Gauto, 1997) tamaño del individuo (Sist \& Nguyen-thé, 2002), el suelo (Clark et al., 1999), siendo éste último pocas veces considerado en estudios de la región y a los daños ocasionados en la masa remanente (Bulfe et al., 2009; Rivero et al., 2008).

\section{Estructura y dinámica}

Los parámetros estructurales del bosque en cuestión son presentados como proporciones de individuos por clase diamétrica al inicio (1998) y al final del período (2006), en claros y parcelas testigo. En ambas situaciones las distribuciones presentaron forma de $\mathrm{J}$ invertida siendo más marcada en parcelas testigo. La frecuencia total en cada situación fue de 167 y 156 ind ha ${ }^{-1}$ para el área de claros en los años 1998 y 2006 respectivamente y de 262 y 295 ind ha ${ }^{-1}$ para el mismo período en parcelas testigo. En cada tratamiento de cosecha las distribuciones presentan también forma de J invertida, siendo para CIR de apariencia más próxima a la determinada para las parcelas testigo.

En valores relativos, referidos al total de individuos, el ingreso fue superior $(27 \%$ ) y la mortalidad inferior (13\%) en las parcelas testigo, presentando un comportamiento inverso el área de claro (20\% y 23\%, respectivamente), siendo estos coincidentes con los resultados obtenidos por Teresczcuch et al. (2007), los cuales compararon áreas aprovechadas bajo diferentes metodologías (convencional y de impacto reducido) y a su vez éstas con áreas sin intervención. Por otro lado, Sist \& Nguyenthé (2002) determinaron un mayor número de ingresos en la medida que la intensidad de cosecha aumenta, con valores de 14 a 33 ind ha ${ }^{-1}$ para las diferentes intensidades de cosecha, siendo contrario a los resultados de este trabajo.

El incremento diamétrico acumulado para el total de los individuos en parcelas testigo y claros fueron de 176,8 y $248 \mathrm{~cm}_{\text {año }}{ }^{-1}$, respectivamente. El incremento diamétrico acumulado únicamente de los ingresos, durante el período 1998-2006, fue de $67 \mathrm{~cm}_{\text {año }}{ }^{-1}$ en 
claros y $55 \mathrm{~cm}$ año ${ }^{-1}$ en parcelas testigo, siendo en valores relativos referidos al total de individuos en estudio de $27 \%$ y $31 \%$ del incremento total (Figura 2).

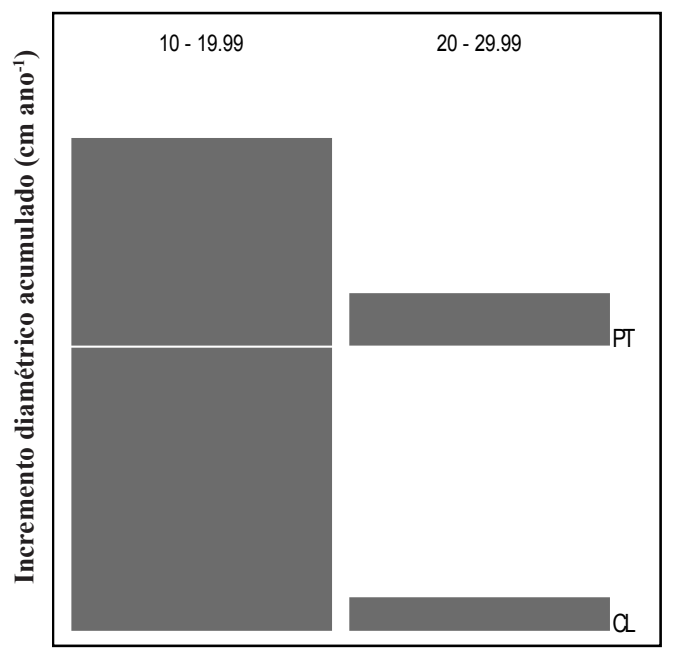

Figura 2. Suma del incremento diamétrico $\left(\mathrm{cm} \mathrm{año}^{-1}\right)$ para el período 1998-2006, por clase de diámetro en el reclutamiento de las especies, en cada situación de estudio. PT: parcelas testigo, CL: claros de corta.

En las frecuencias de los individuos por clase de incremento diamétrico en áreas de claros y parcelas testigo se observa la tendencia de muchos individuos en la población con crecimientos bajos. Sin embargo, las áreas de claros presentaron una cantidad superior de individuos con crecimientos mayores a $0,4 \mathrm{~cm}_{\text {año }}{ }^{-1}$. En relación con el número de individuos en cada situación de estudio, en el área de claro es inferior, por lo tanto, el mayor incremento diamétrico acumulado estaría relacionado principalmente a la mayor abundancia de individuos con altos crecimientos. Estos altos crecimientos estarían asociados principalmente a las especies de las clases diamétricas inferiores (Rivero et al., 2008).

Una proporción superior del crecimiento en áreas testigo es dada por el ingreso, mientras que para el área de claro el crecimiento estaría más relacionado con los individuos ya establecidos en el período de estudio. Un análisis conjunto del ingreso y del incremento diamétrico acumulado por estos individuos hacen notar su mejor desempeño en áreas de claro, siendo que aquí el ingreso es inferior y la suma del incremento diamétrico superior.

\section{Conclusiones y recomendaciones}

El aprovechamiento de impacto reducido podría ser una alternativa para las extensas áreas de bosques nativos sometidas a manejo en la región de estudio. Esta técnica debería ser considerada como menos agresiva cuando comparada a las técnicas usuales de cosecha desarrollada en la zona. Dadas las características de la dinámica del bosque estudiado, y la particularidad de tener una colonización masiva por bambúseas, se resalta la necesidad de la aplicación de tratamientos silviculturales post-cosecha para obtener mejores resultados en la estructura del bosque además, de los ya obtenidos con la aplicación de las técnicas de impacto reducido.

El aprovechamiento planificado favorece el crecimiento de los remanentes y la estructura del bosque se observa menos perturbada cuando comparada con un bosque bajo manejo tradicional de cosecha.

El análisis del crecimiento diamétrico por grupos ecológicos permitió una mayor homogenización del mismo, facilitando la interpretación de los datos. En bosques con alta diversidad florística, se debería considerar a los grupos ecológicos como una herramienta base para el manejo y conservación de los mismos. Debido a la dificultad encontrada para clasificar a las especies en los diferentes grupos ecológicos, por la carencia de datos tanto morfológicos como ecológicos/ fisiológicos de las especies, sería interesante incursionar en estos aspectos a nivel de especies para obtener información más calificada.

Sería interesante incorporar a este tipo de estudios otras formas de vida tales como herbáceas, epífitas y lianas, además de la fauna asociada a éstas, para obtener una conclusión más global de la dinámica del ecosistema en estudio y poder llegar a una mayor aproximación del "teórico" manejo sustentable.

\section{Agradecimientos}

A CIFOR (Center International Forestry Research) por el financiamiento y a la Facultad de Ciencias Forestales (UNaM) por el apoyo logístico para la realización de este trabajo. 


\section{Referencias}

BULFE, N.; GALVÃO, F.; FIGUEIREDO FILHO, A; MAC DONAGH, P. Efeitos da exploração convencional e de impacto reduzido em uma floresta estacional semidecidual na provincia de Misiones, nordeste da Argentina. Revista Floresta, v. 39, n. 2, p. 365-379, 2009.

BUDOWSKI, G. Distribution of tropical American rain forest species in the light of succesional processes. Turrialba, n. 15, n. 1, p. 40-42, 1965.

CAMPANELLO, P. Diversidad, crecimiento y fisiología de árboles en la selva Misionera: efectos de los cambios en la estructura y funcionamiento del ecosistema producidos por la invasión de lianas y bambúseas. 2004. 153 p. Tesis (Doctorado en Ciencias Biológicas) - Universidad de Buenos Aires, Argentina.

CARVALHO, P. E. R. Espécies arbóreas brasileiras. Brasília, DF: Embrapa Informação Tecnológica; Colombo: Embrapa Florestas, 2003-2008. 3 v. (Coleção espécies arbóreas brasileiras, v. 1-3)

CLARK, D. B.; PALMER, N. W.; CLARK, D. A. Edaphic factors and the landscape-scale distribution of tropical rain forest trees.

Ecology, v. 8, n. 80, p. 2662-2675, 1999.

CONNELL, J. Diversity in tropical rain forests and coral reefs. Science, v. 199, p. 1302-1309, 1978.

DENSLOW, J. S. Gap partitioning among tropical rainforest trees. Biotropica, v. 12, n. 2, supl., p. 47-55, 1980.

EIBL, B.; GONSESKI, D.; BOBADILLA, A.; SILVA, F.; WEBER, E.; ARTHUS, H. Boletín Agrometeorológico. Año 7, n. 1. Enero - Diciembre 1995. Eldorado: UNaM, 1995.

FERREIRA LIMA, R. A. Estrutura e regeneração de clareiras em Florestas Pluviais Tropicais. Revista Brasileira de Botânica, v. 28, p. 651-670, 2005.

FINEGAN, B. Los gremios de especies forestales. In: FINEGAN, B. Bases ecológicas para la silvicultura. CATIE. Turrialba, Costa Rica. 1993. p ?-?.

GAUTO, O. Análise da dinâmica e impactos da exploração sobre o estoque remanescente (por espécie e por grupos de espécies similares) de uma Floresta Estacional Semidecidual em Misiones, Argentina. 1997. 93 p. Dissertação (Mestrado em Ciências Florestais) - Universidade Federal do Paraná, Curitiba.

GUZMÁN G., R. A. Consideraciones teóricas y metodologías prácticas para la asignación de gremios ecológicos para las especies forestales de bosque húmedos tropicales. Santa Cruz: BOLFOR, 1997. 21 p. (BOLFOR. Documento técnico, 58)

HOGAN, K. P; MACHADO, J. L. La luz solar: consecuencias biológicas y su medición. In: GUARIGUATA, M; KATTAN, G. Ecología y Conservación de Bosques Neotropicales. Costa Rica: Libro Universitario Regional, 2002, 692 p.

LOUMAN, B.; QUIRÓS, D.; NILSSON, M. (eds). Silvicultura de bosques latifoliados húmedos con énfasis en América Central. Turrialba: CATIE. 2001, 265 p. (CATIE. Serie técnica. Manual técnico, 46.)
MAC DONAGH, P.; GARIBALDI, J.; RIVERO, L.; SNOOK, L.; TOMA, T. Comparación de daños, mortalidad y reclutamiento en dos sistemas de aprovechamiento forestal en Misiones, Argentina. In: JORNADAS TECNICAS FORESTALES Y AMBIENTALES, 10., 1989, Eldorado. Actas... Eldorado: FCF-UNaM-INTA. 2003. p. ?-?

MARTINS-MACIEL, M. N.; FARINHA WATZLAWICK, L.; SCHOENINGER, E. R.;

MINORU YAMAJI, F. Efeito da radiação solar na dinâmica de uma floresta. Revista Ciências Exatas e Naturais, Brasil, v. 4, n. 1, p. 101-114, 2002.

PAHR, N.; FERNÁNDEZ, R.; O'LERY, H.; LUPI, A.

Relevamiento edafológico del Área Experimental y

Demostrativa "Guaraní" de la Facultad de Ciencias Forestales (UNaM) -Escala 1:50.000-. Eldorado, Misiones: UNaM, 1997,

$39 \mathrm{p}$.

PALAVECINO, J.; MAIOCCO, D. Levantamiento del medio físico del área de investigación forestal Guaraní, Misiones. Yvyraretá, v. 6, p. 50-63, 1995.

RIVERO, L.; MAC DONAGH, P.; GARIBALDI, J.; TOMA, T. CUBBAGE, F. Impacts of conventional and reduced impacts logging on growth and stand composition four years after harvest in a neotropical forest in Misiones, Argetina. Scientia Forestalis, Piracicaba, v. 36, n. 37, p. 21-31, 2008.

SCATENA, F.N. El bosque neotropical desde una perspectiva jerárquica, In: GUARIGUATA, M.; CATAN, G. Ecología y Conservación de Bosques Neotropicales. Costa Rica: Libro Universitario Regional, 2002. p. 23-41

SIST, P.; NGUYEN-THÉ, N. Logging damage and the subsequent dynamics of a dipterocarps forest in East Kalimantian (19901996). Forest Ecology and Management, v. 165, p. 85-103, 2002.

SIST, P.; FERREIRA, F. N. Sustainability of reducedimpact logging in the Eastern Amazon. Forest Ecology and Management, v. 243, p. 199-209, 2007.

TERESCZCUCH, M.; MAC DONAGH, P.; DE OLIVEIRA, A.; RIVERO, L.; BULFE, N. Predicción de la estructura diamétrica de especies comerciales de un bosque subtropical por medio de matrices de transición. Floresta. v. 37, n. 1, p. 71-82, 2007.

TRESSENS, S.; REVILLA, V. Riqueza florística de un bosque nativo de 5000 hectáreas conducido racionalmente en la provincia de Misiones. In: CONGRESO ARGENTINO Y LATINOAMERICANO, 2, 1997, Posadas. Anales... Posadas: AFoA, 1997.

UHL, C.; CLARK, K.; DEZZEO, N.; MAQUIRINO, P.

Vegetation dynamics in Amazonian treefall gaps. Ecology, v. 69, p. 751-763, 1988.

SWAINE, M. D.; WHITMORE, T. C. On the definition of the ecological species groups in tropical rain forest. Vegetatio, n. 75, p. 81-86, 1988.

WHITMORE, T. C. Tropical rain forest of the Far East. 2 ed. Oxford Science. 1984, 352 p. 